\section{- Prof. Dr.-Ing. Karl-Friedrich Thöne (1956-2016) verstorben DVW verliert seinen Präsidenten}

Das Präsidium des DVW gibt bekannt:

Tief bestürzt und betroffen haben wir erfahren, dass der Präsident des DVW e.V., Prof. Dr.-Ing. Karl-Friedrich Thöne, kurz vor seinem 60. Geburtstag überraschend verstorben ist. Wir verlieren einen großen Geodäten, einen geschätzten Menschen und einen außergewöhnlichen Freund. Unser aufrichtiges Mitgefühl gilt seiner Frau und seiner Familie.

Wir werden ihm ein ehrendes Andenken bewahren.

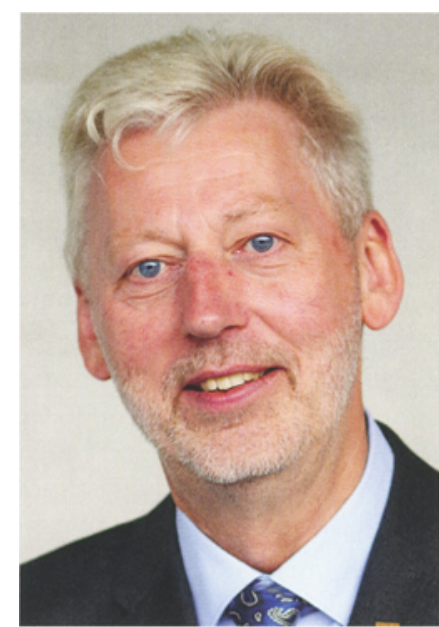

Die Nachricht erreichte uns kurz vor Redaktionsschluss dieser Ausgabe. In den nächsten KN wird ein Nachruf auf KarlFriedrich Thöne abgedruckt.

\title{
Rezensionen
}

Bandarova, Temenoujka; Konecny, Milan; Zlatanova, Sisi (Hrsg.):

\section{Thematic Cartography for the Society}

Springer-Verlag Cham, Heidelberg, New York, Dordrecht, London 2014, 357 S. 131 schwarz-weiß Abb., e-Book: ISBN 978-3-319-08180-9, $83,29 €$ (einzelne Beiträge je $29,69 €)$; gebunden: ISBN 978-3-319-08179-3 106,99 €.

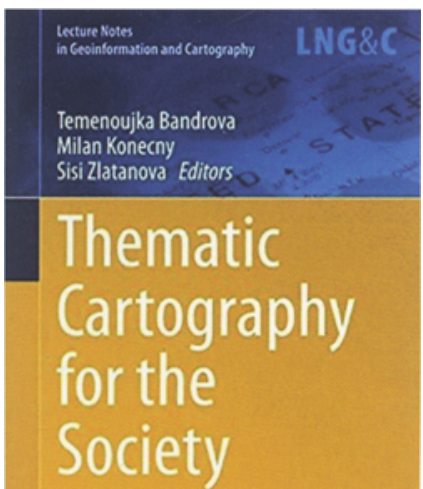

kurze Information gegeben werden. Dabei handelt es sich um die $5^{\text {th }}$ Jubilee International Conference on Cartography and GIS (Seminar with EU cooperation on Early Warning and Disaster/Crisis Management), die von der Bulgarischen Kartographischen Gesellschaft, der Internationalen Kartographischen Vereinigung (IKV) und von der Universität für Architektur, Bauingenieurwesen und Geodäsie (Sofia) organisiert wurde. An der Konferenz nahmen 170 Fachleute aus 42 Staaten teil. Wegen einer Überschwemmungs-Katastrophe mussten die Teilnehmer nach dem Abschluss der Konferenz mit Hubschraubern gerettet werden. In der vorliegenden Publikation wurden die 30 besten Vorträge abgedruckt. Erfreulicherweise sind hier Fachkollegen auch aus solchen Staaten präsent, wie z. B. aus Saudi-Arabien, Ägypten, Indien und Indonesien, aus denen meist nur spärliche Informationen bezüglich Kartographie und GIS vorliegen. Die anderen Vorträge wurden nicht im Druck, sondern im Internet veröffentlicht: http://cartography-gis. com/docsbca/5ICCandGIS_ Proceedings.pdf. Mit dem Entstehungszusammenhang der Studien lässt sich erklären, dass sie thematisch etwas zu divergente Vielfalt aufweisen. Den Herausgebern gelang es jedoch, durch die Bildung von fünf Themengruppen die heterogenen Themen zu strukturieren.

Die erste Themengruppe befasst sich mit benutzerfreundlichem Internet und Web-Kartographie. Die erste Studie weist die größte Konformität zu den Intentionen der Herausgeber auf: um die Qualität der Internetkarten zu heben, benötigen Nutzer leicht anwendbare Möglichkeiten zur Analyse von räumlichen Daten und zur ihrer kartographischen Visualisierung. In der Studie über Open-Data-Platform und Map Design (S. 3-11) zeigen T. Mildorf, J. Jezek, O. Cerba (Pilsen), Ch. Malewski, S. Templer (Darmstadt), M. Sredl und K. Charvat
(Benešov) am Beispiel europäischer Landnutzungsdaten, wie man große Datenmengen aus verschiedenen Provenienzen integrieren kann und in thematischen Karten, unter Beibehaltung kartographischer Prinzipien unter Verwendung von Open-SourceWerkzeugen, wiedergeben kann. Die Harmonisierung, d. h. die Integration heterogener Datenmodelle, erfolgte durch den Humboldt Alignment Editor, der durch ein europäisches Forschungsrahmenprogramm erarbeitet wurde. Bedauerlicherweise enthält der Beitrag keine Kartenbeispiele. N. Baranovskiy (Tomsk) und M. Zharikova (Kherson) entwickelten ein Modell für die Beurteilung von möglichen Gefahren von Waldbränden in der Ukraine (S. 13-22). Dieses Modell wurde dann für ein Geoinformationssystem zugrunde gelegt. Ebenfalls mit Geoinformationssystemen erstellten A. A. Solyma, M. M. El-Hattab, R. I. Rifai (Sadat City) und Waleed M. T. Fakbar (Kairo) Karten zur unterschiedlichen Gefahrenszenarien, die durch Steigung des Meeresspiegels an der Nordküste von Ägypten entstehen könnten (S. 23-31). Schließlich beschreiben S. Meier, F. Heidmann und A. Thom (S. 33-44) einen neuen Ansatz für die Entwicklung von Echtzeit-Heatmaps (Dichtekarten) für mobile Geräte, die „Heattile“ genannt werden. Es erfolgt hier ein serverseitiges Pre-Clustering, wobei das Koordinatensystem, das auf der Mercator-Projektion basiert, in kleine Quadrate aufgeteilt wird. Dieses Verfahren hat bestimmte Vorteile bezüglich der Interaktionen, aber auch einige Nachteile durch die begrenzten Visualisierungsmöglichkeiten.

Die Studien des zweiten Themenbereiches befassen sich mit der benutzerorientierten Kartierung. A. Dukaczewski (Warschau) berichtet über seine Untersuchungen bezüglich der Perzeption von einfachen und komplexen dynamischen Karten nach einzelnen Altersgruppen (S. 47-60). Die statistischen Auswertungen zei- 